\title{
Plastic Surgery for Pressure Sores
}

Michael Pers, M.D.

The Department of Plastic Surgery, Rigshospitalet, Copenhagen, Denmark

Plastic surgery can offer surgical reconstruction following excision of pressure sores in paraplegic patients. Before surgical treatment is initiated it should, however, be borne in mind that satisfactory primary results are difficult to achieve regularly, and that long-term results will sometimes be disappointing.

\section{Focus on bleeding and infection}

In order to pinpoint the factors of importance for the improvement of the initial results of surgical closure of pressure sores, an analysis of 103 consecutive cases was undertaken (Pers et al., 1986). It was concluded that hematomas and infection were the main cause of failure. Consequently it was decided to focus on the control of secondary bleeding and infection. Decubital ulcers being contaminated, it is obvious that hematomas invite infection.

\section{Preoperative measures}

Prior to surgery the patient should be in good general condition. If not, a nutritious diet, including supplementary protein, minerals and vitamins, should be provided. The wound should be cleansed daily with soap and water and covered with a large absorbent pad, and changed as required. No dressing material should be put into the wound cavity. From the very beginning of the treatment the patient's bed should be provided with a water mattress.

\section{First operation: radical excision}

To control bleeding and infection it is helpful to perform surgery in two stages. The first operation is a radical excision of infected scar tissues. The granulating wound surface is dyed with methylene blue, and all coloured tissue is removed. One or two samples of tissue are sent for bacteriological tests so that the appropriate antibiotics can be used at the time of reconstruction. Exposed bone is removed with a chisel, but no major reduction of bony prominences is aimed at. After haemostasis the wound is loosely packed with Iodoform gauze and the patient is placed prone in bed. 


\section{Second operation: reconstruction}

Seven days later the wound will be in the proliferative phase, ready to receive a vascularised muscle flap. Depending on the location of the defect one of three procedures can be recommended:

Ischial sores. Sores located over the ischial tuberosity can be closed after the mobilisation of a triangular musculocutaneous island flap based on the hamstring muscles on the back of the thigh (Hurteau et al., 1981). Conserving the vessels and continuity between the skin and the underlying muscles, the muscles are freed from their fibrous connections and gradually cut, until the flap slides upwards to fill the defect, without any tension. In principle the procedure is a V-Y plasty (Fig. 1).

Sacral sores. For closure of defects in the sacral region, the upper part of the gluteus maximus muscle is mobilised on a medially based pedicle, (Maruyama

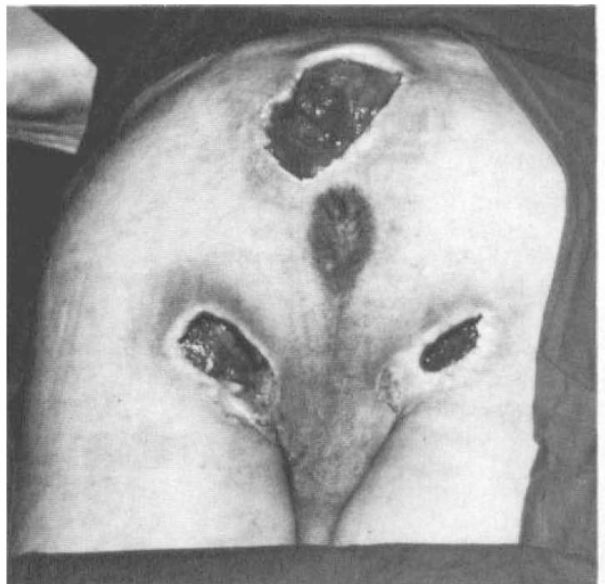

A

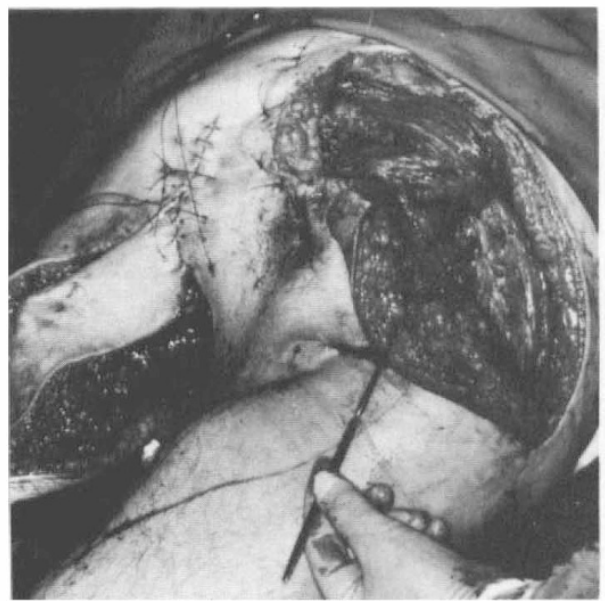

C

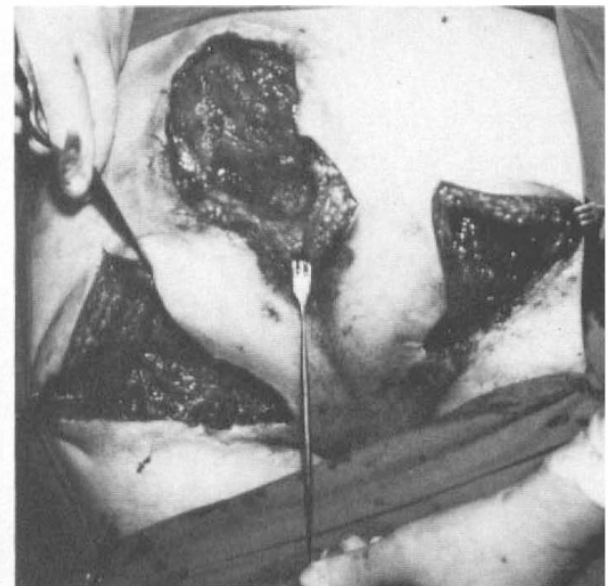

B

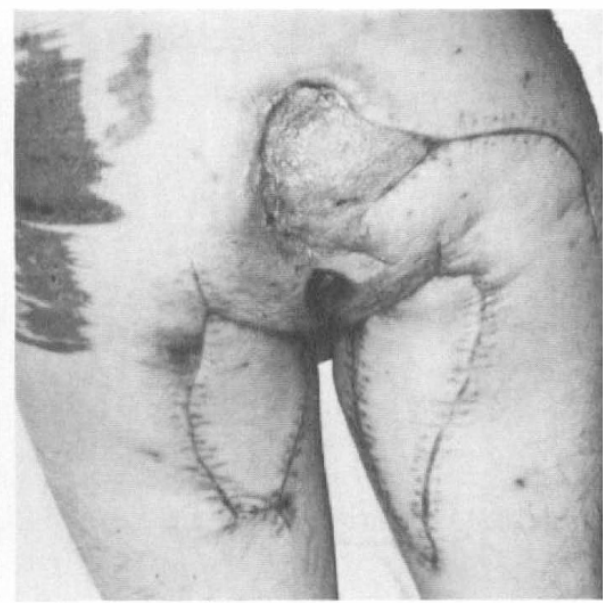

D

Figure 1 (A) Sacral and ischial pressure sores ready for surgical treatment. (B) First stage: complete excision and hemostasis. (C) Second stage 1 week later: the gluteus maximus muscle is turned over and the hamstring muscles with overlying skin are advanced upwards. (D) The muscle in the sacral region was covered with a split thickness skin graft. Result after 4 weeks. 
et al., 1980). The muscle flap is hinged over its pedicle to be placed inside-out in the defect. The exposed muscle is covered with a split skin graft (Fig. 1). This manoeuvre is preferable to the musculocutaneous gluteus maximus island flap procedure, because the secondary defect can be closed without tension, while tension might well become a problem after the excision of a large skin island.

Trochanteric sores. Exposed bone can be covered with a musculocutaneous tensor fascia lata flap (Hill et al., 1978), which is conveniently located just in front of a trochanteric sore (Fig. 2). The flap has a proximal pedicle, which includes muscle, blood vessels and overlying skin. If needed, the flap may be extended as far as the middle of the thigh, provided the fascia is included. The secondary defect is covered with a split skin graft.

\section{Drainage}

Following the excision the wounds are left open. Blood and lymphatic secretion will be absorbed by Iodoform gauze and a bulky dressing, which should be changed before it is soaked through. After the reconstruction, when the defect has been closed, oozing of blood will only be seen from the tissue where the muscle flap has been cut and not from the primary defect. Two suction drains should be inserted, one under the muscle flap, and one in the secondary defect, which is closed with $2 / 0$ nylon sutures on large needles, picking up the floor of the wound.

\section{Fibrin sealant}

In order to obtain haemostasis and close adhesion of the tissues, fibrin sealant can be used. Application of 1 or 2 millilitres of fibrinogen-thrombin mixture can be facilitated by mixing the two components in a jet of compressed air (Fig. 3). A coagulation time of 3 minutes is achieved by adding 4 units of thrombin to 1 millilitre of fibrinogen.

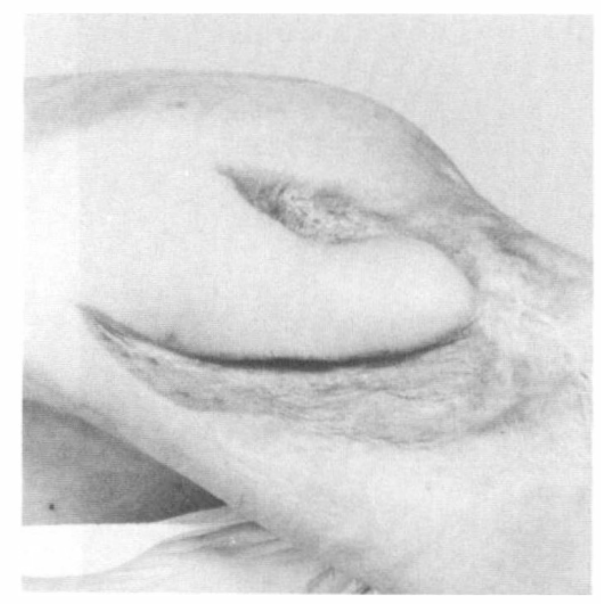

Figure 2 Tensor fascia lata flap covering trochanteric pressure sore.

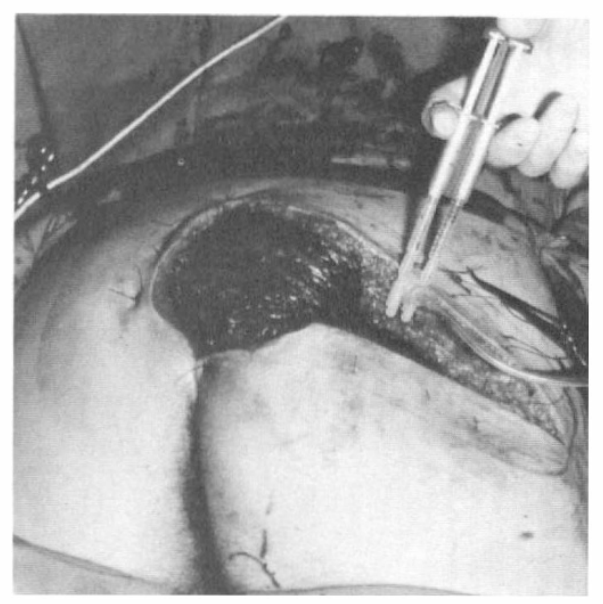

Figure 3 Application of fibrin sealant (Tisseel). The two components are mixed in a jet of compressed air. 


\section{Antibiotics}

There will be no need for antibiotics following the initial excision, but on the morning of the wound closure, a full dose of antibiotics, in accordance with sensitivity, is given for 2 days.

\section{Postoperative care}

Drains should be left in situ until the secretion is less than 5 millitres a day, even if this takes 1 or 2 weeks. As a rule the patient should be nursed in a prone position for 10 to 14 days, thereafter in a supine position, but confined to bed for another 2 weeks. Four weeks after the second operation the patient can be allowed to sit up in a well padded wheel-chair for a few hours at adequate intervals. At this point it is important that the patient has been trained to lift himself up by his arms every 20 minutes and that exposure of the repaired region to shearing forces is avoided, i.e. the patient should not be moved without being lifted.

\section{Conclusion}

Pressure sores in paraplegic patients can be cured by reconstructive surgery as outlined above. Such treatment usually takes about 6 weeks. It does not protect the patient from developing a similar sore again. Whenever a sore is repaired a thorough investigation should be made to throw light on the causes and mechanisms of the pressure, and measures should be taken to prevent the patient from exposure to a similar risk in the future.

\section{References}

Hill LH, Nahai F, Et Vasconez LO 1978 The tensor fascia lata myocutaneous free flap. Plastic Reconstructive Surgery 61:517

HuRTeAu JE, Bostwick J, NAHAi F, et al., $1981 \mathrm{~V}-\mathrm{Y}$ advancement of hamstring musculocutaneous flap for coverage of ischial pressure sore. Plastic Reconstructive Surgery 68:539-542

MaruYama Y, Nakajima H, Wada M, et al., 1980 A gluteus maximus island flap for the repair of a sacral decubitus ulcer. British fournal of Plastic Surgery 33:150-155

Pers M, SNORRASON K, Nielsen IM 1986 Primary results following surgical treatment of pressure sores. Scandinavian Fournal of Plastic Reconstructive Surgery 20:123-124 